\title{
Previsão da demanda como suporte à filosofia lean
}

\section{Demand forecast as support for the lean philosophy}

Pedro Vieira Souza Santos ${ }^{1}$

${ }^{1}$ Engenheiro de Produção, Universidade Federal do Vale do São Francisco - UNIVASF. Petrolina - PE, Brasil. pedrovieirass@hotmail.com

\begin{abstract}
Resumo
O pensamento lean pode ser traduzido como um conjunto de princípios, ferramentas e técnicas que, combinadas, objetivam a melhoria, em termos de qualidade, custo, satisfação do cliente e outros. Diversas ferramentas e métodos podem auxiliar as organizações na busca por um ambiente fabril "enxuto", desde por exemplo ferramentas da qualidade, tecnologia da informação e ainda, métodos matemático-estatísticos, como a previsão da demanda. Assim, o presente artigo que comporta-se como estudo de caso, teve como objetivo analisar a aplicação do modelo de Holt-Winters para Efeitos Sazonais Aditivos, numa empresa fabricante de polpa de frutas localizada em Pernambuco e comparar os ganhos obtidos sob a ótica da filosofia lean. Observou-se, entre outros ganhos, menor nível de estoques, tempo de espera reduzido e ainda menores custos. Ou seja, comprova-se que, quando aplicada a técnica de previsão da demanda, a gestão da empresa pode planejar melhor suas aquisições, determinar investimentos em ativos, contratação de mão de obra, ampliação da capacidade produtiva e ainda, estimar lucro da mesma.
\end{abstract}

Palavras-chave: Gestão. Indústria. Lean Manufacturing.

\begin{abstract}
Lean thinking can be translated as a set of principles, tools and techniques that, combined, aim for improvement in terms of quality, cost, customer satisfaction and others. Several tools and methods can assist organizations in the quest for a "lean" manufacturing environment, for example quality tools, information technology and mathematical-statistical methods such as demand forecasting. Thus, the present article, which behaves as a case study, aimed to analyze the application of the Holt-Winters Model for Seasonal Effects in a fruit pulp company located in Pernambuco and compare the gains obtained from the perspective of the lean philosophy. Among other gains were observed lower inventory levels, reduced waiting time and even lower costs. That is, it is proven that, when applied to the demand forecasting technique, the company's management can better plan its acquisitions, determine investments in assets, hiring labor, increase production capacity and estimate its profit.
\end{abstract}

Keywords: Management. Industry. Lean Manufacturing. 


\section{Introdução}

Conforme a evolução do mercado, com aumento da competitividade, as organizações são induzidas a buscarem, cada vez mais, métodos que proporcionem melhorias em suas operações e processos que as conduzam para a sustentabilidade dos negócios (Santos \& Lima, 2018; Santos, 2017). Uma das práticas observadas é a manufatura enxuta ou lean manufacturing que auxilia no uso adequado de insumos, otimização de recursos e métodos de manufatura (Wilson, 2010).

O pensamento lean, traduz-se como um conjunto de princípios, ferramentas e técnicas que, combinadas, objetivam a melhoria, em termos de qualidade, custo e satisfação do cliente e outros, eliminando operações e/ou atividades que não agregam valor ao produto (Drew, McCallum \& Roggenhofer, 2016). Esta filosofia, tem como foco a redução de desperdícios inerentes ao ambiente interno da organização, isto é, em qualquer área da mesma, buscando otimizar recursos principais, onde, de acordo com Bayat \& Dadashzadeh (2017) em qualquer tipo de indústria tem um impacto direto e positivo nos processos de manufatura.

Logo, pode-se citar diversos benefícios oriundos da prática lean nas empresas. São vantagens relacionadas a melhoria da qualidade do produto, redução do tempo de ciclo, entregas no prazo adequado, aumento do lucro, redução de custos, melhor utilização de mão de obra, redução de estoques, maior flexibilidade, melhor utilização do espaço e ainda, maior produtividade local (Panizzolo, Garengo, Sharma \& Gore, 2012). Isto posto, nota-se que o pensamento enxuto é uma cultura de busca pela otimização, real e contínua. Ademais, para Sabadka, Molnar \& Fedorko (2017), deve ser praticado continuamente como uma missão organizacional de longo prazo com ganhos significativos.

Ultimamente, o lean tem sido difundido em diversos ambientes, encontrando-se em aplicação em todo mundo em setores diversos, como serviços, empreendedorismo, tecnologia da informação, confiabilidade e, principalmente, no ambiente industrial (Alefari, Salonitis \& Xu, 2017). Além disso, diversas ferramentas e métodos podem auxiliar as organizações na busca por um ambiente fabril "enxuto", desde por exemplo ferramentas da qualidade, tecnologia da informação e ainda, métodos matemático-estatísticos, como a previsão da demanda.

Para Klassen \& Flores (2001), a previsão de demanda é um processo capaz de fornecer, com alto grau de confiabilidade, informações relevantes sobre o comportamento pontual dos produtos ao longo do tempo. Sua importância pauta-se no fato de que é fundamental para a tomada de decisão, desde o planejamento de capacidade, programação de estoque, melhorias internas e planejamento da produção.

Há, portanto, diversos tipos de métodos de previsão de demanda, desde modelos qualitativos a quantitativos. As técnica qualitativas que abordam a previsão levam em consideração, em geral, 
opiniões, experiência e julgamentos de especialistas (Slack, Jones \& Johnston, 2009). Por outro lado, de acordo com Lee, Song \& Mjelde (2008), os métodos do tipo quantitativos apresentam-se com base em uma variedade de métodos estatísticos, com diferentes particularidades e níveis de complexidade. Um deles é o método de Holt-Winters para Efeitos Sazonais Aditivos, aplicado em séries temporais.

Este tipo de método, orientado por séries temporais, apresentam a ideia de que as observações passadas da série possuem informações significativas sobre o seu padrão de comportamento no futuro. A natureza desse modelo, consiste basicamente em identificar o padrão da série, considerando erros possíveis intrínsecos as observações individuais, e utilizá-lo para prever os valores futuros (Henning, Alves \& Konrath, 2010).

Assim, o presente artigo tem como objetivo analisar a aplicação do modelo quantitativo de previsão de demanda, intitulado método de Holt-Winters para Efeitos Sazonais Aditivos, numa empresa fabricante de polpa de frutas localizada em Pernambuco e comparar os ganhos obtidos sob a ótica da filosofia lean.

\section{Revisão da literatura}

\subsection{Filosofia Lean}

O termo lean foi introduzido ao final dos anos 80 , resultado do estudo sobre manufatura automobilística japonesa. Trata-se de uma nomenclatura criada pelos pesquisadores do Massachussets Institute of Technology (Begam, Swamynathan \& Sekkizhar, 2014). A definição se concentra na eliminação de desperdícios, ou seja, no que não agrega valor ao produto manufaturado (Pajak, 2013). Além disso, sua implementação é eficiente e reduz custos operacionais da empresa, aumentando a vantagem competitiva (Trojanowska, Kolińska \& Koliński, 2011).

O lean é entendido, em suma, como uma estratégia para alcançar uma melhoria contínua significativa no desempenho da organização. Evoluiu a partir do Sistema Toyota de Produção (STP) depois da Segunda Guerra Mundial como um novo modelo de negócios, influenciado pela escassez de recursos no Japão, em contraste com a vasta quantidade de insumos disponíveis para manufatura nos EUA (Gobinath, Elangovan \& Dharmalingam, 2015).

A aplicação dos princípios e ferramentas da filosofia lean são difundidas, crescentemente, em diversos setores (Lopes, Freitas \& Sousa, 2015), resultado do reconhecimento de que as organizações que atuam de acordo com os métodos de manufatura enxuta, apresentam vantagens relevantes em relação a custos e qualidade, comparando-se com as empresas que adotam ainda a produção em massa tradicional (Forbes \& Ahmed, 2010). Isto é possível devido, entre outros fatores, pelo intuito da filosofia, que torna as operações mais eficientes e econômicas (Dennis, 2016). 
Isto posto, como o lean visa eliminar perdas ao longo do processo, Slack et al. (2013) classificam estes desperdícios em sete tipos:
a) Produção excessiva;
b) Espera;
c) Transporte;
d) Processos desnecessários;
e) Estoque;
f) Movimentação;
g) Defeitos ou falhas no produto/serviço.

Nesse sentido, Zakarial et al. (2017) descrevem, de forma sucinta, cada um dos desperdícios, conforme Quadro 1.

Quadro 1 - Os sete tipos de desperdícios

\begin{tabular}{|c|l|}
\hline Produção em excesso & $\begin{array}{l}\text { O progresso de um produto, um procedimento ou uma competência de } \\
\text { produção sem valor agregado }\end{array}$ \\
\hline Espera & $\begin{array}{l}\text { Períodos longos de ociosidade e/ou pausas de produtos são valores } \\
\text { não agregados ao item. }\end{array}$ \\
\hline Transporte & $\begin{array}{l}\text { Transportando o produto para vários lugares. } \\
\text { Enquanto o produto está em movimento, está em uma situação que } \\
\text { não será processado e, portanto, não conta valores para o cliente. }\end{array}$ \\
\hline Alto Processamento & Traduzido por processar mais que o necessário. \\
\hline Estoque & $\begin{array}{l}\text { Alto nível de armazenamento, tanto de matéria prima quanto de } \\
\text { produtos acabados, gerando custos em excesso. }\end{array}$ \\
\hline Defeitos & $\begin{array}{c}\text { Deslocamento de operador sem necessidade, não dando apoio ao } \\
\text { processo. }\end{array}$ \\
\hline Movimentação & Falhas na qualidade do produto, gerando produtos defeituosos, \\
\hline exindo retrabalho.
\end{tabular}

Fonte: Adaptado de Zakarial et al. (2017) 
Ademais, o Lean requer uma melhoria contínua que possibilite a manutenção de práticas enxutas (Władysiak, 2006), baseado no fato de que o objetivo é limitar os tipos de desperdício em todas as áreas do empresa (Sokovic, Pavletic \& Fakin, 2005). Assim, a manufatura adaptada segundo os conceitos lean, na visão de Modi \& Thakkar (2014) podem apresentar vários benefícios, como:

\footnotetext{
$\checkmark$ Redução de custo;

$\checkmark$ Redução no lead time;

$\checkmark$ Eliminação de perdas;

$\checkmark$ Melhoria na produtividade;

$\checkmark$ Maior qualidade no processo/operações;

$\checkmark$ Menor tempo de ciclo;

$\checkmark$ Mitigação de atividades desnecessárias;

$\checkmark$ Maior produtividade.
}

Nota-se, portanto, que a filosofia Lean propõe, na prática, combinar as diversas técnicas de gestão com a realidade local, a fim de ter uma produtividade maior com menos recursos possíveis. Envolve ainda mudanças ao longo de todo processo produtivo, considerando ainda relações com fornecedores, projeto e engenharia, organização interna da fábrica e distribuição (Womack, Jones \& Roos, 2004). Shiver \& Eitel (2010) reforçam que a filosofia, por ter origem em processos de manufatura, aperfeiçoa os negócios, tornando-os eficazes.

\subsection{Previsão da demanda}

A previsão da demanda é um dos principais desafios em qualquer negócio, sendo a chave para uma melhor gestão da empresa, com minimização de riscos devido a indisponibilidade de estoque ou ainda com custos menores devido a um inventário melhor planejado. Esta prática, constitui-se como uma previsão estimada por um produto ou lotes destes em uma faixa de tempo, considerando dados de entrada como registros históricos de vendas (Bose, Flunkert, Gasthaus \& Januschowski, 2017).

Determinar comportamento futuro, em termos de previsão é um auxílio na tomada de decisão, desempenhando um papel importante na gestão (Hyndman, Koehler, Ord \& Snyder, 2008). "O planejamento e controle das atividades dependem de estimativas acuradas dos volumes de produtos e serviços a serem processados. Tais estimativas ocorrem tipicamente na forma de planejamento e previsões" (Ballou, 2006, p. 241). 
Nesse sentido, diversas opções podem ser consultadas com o intuito de realizar a previsão (Kerkkänen, Korpela \& Huiskonen 2009). Os métodos de previsão podem ser baseados em modelos matemáticos (que usam os dados históricos disponíveis), em métodos do tipo qualitativos (que aproveitam a experiência gerencial) ou ainda numa combinação de ambos (Ritzman \& Krajewski, 2004).

Lustosa, Mesquita, Quelhas \& Oliveira (2008) citam alguns tipos principais de métodos de previsão de demanda, sendo:

$\checkmark$ Qualitativos: Método Delphi, pesquisa de mercado, analogia histórica, simulação de cenários;

Quantitativos: Média móvel, suavização exponencial, análise de regressão, modelos econométricos.

Para Corrêa, Gianesi \& Caon (2012), os métodos de previsão de demanda classificados como qualitativos, são caracterizados com base em opiniões e julgamentos pessoais, ou seja, uma análise mais subjetiva do contexto em análise. Para Moreira (2001), esses métodos consideram o julgamento e a experiência de especialistas da empresa. Sendo, de acordo com o autor, utilizadas quando não há dados disponíveis ou ainda quando os dados históricos são insuficientes para a modelagem matemática.

Por outro lado, de acordo com Makridakis, Wheelwright \& Hyndman (1998), o emprego de métodos quantitativos, baseado em métodos matemáticos depende basicamente do comportamento da série temporal que se deseja analisar, baseando-se em dados quantitativos e técnicas estatísticas. Dentre essa gama de metodologias de cunho matemático, um dos métodos empregados para as séries de dados históricos, que apresentem tendência e sazonalidade aditiva, sendo também o modelo mais eficiente é o denominado aditivo de Holt-Winters (Koehler, Snyder \& Ord, 2001).

\subsection{Método de Holt-Winter para efeitos sazonais aditivos}

O modelo classificado como sazonal aditivo é utilizado quando os dados comportam-se com uma amplitude de variação sazonal constante ao longo dos períodos observados de tempo. Este modelo é representado pelas seguintes equações matemáticas (Makridakis et al., 1998):

$$
\begin{array}{ll}
\mathrm{Lt}=\alpha(\mathrm{yt}-\mathrm{St}-\mathrm{S})+(1-\alpha)(\mathrm{Lt}-1+\mathrm{Tt}-1) & 0 \leq \alpha \leq 1 \\
\mathrm{Tt}=\beta(\mathrm{Lt}-\mathrm{Lt}-1)+(1-\beta) \mathrm{Tt}-1 & 0 \leq \beta \leq 1 \\
\mathrm{St}=\gamma(\mathrm{yt}-\mathrm{Lt})+(1-\gamma) \mathrm{St}-\mathrm{S} & 0 \leq \nu \leq 1
\end{array}
$$


Para o cálculo, $\alpha, \beta$ e $\gamma$ são as constantes de suavização que estimam o peso relativo ao nível (Lt), a tendência (Tt) e a sazonalidade (St), respectivamente.

A equação de previsão é dada, portanto, por:

$$
Z_{t+k}=L_{t}+{ }_{k} T_{t}+S_{t-s+k}
$$

Onde:

$$
\begin{aligned}
& \text { Lt = nível da série (ou média suavizada) } \\
& \mathrm{Tt} \text { = Tendência } \\
& \mathrm{St} \text { = sazonalidade. }
\end{aligned}
$$

Em suma, pode-se entender que o modelo acima citado é aplicado numa série de dados onde "o efeito sazonal não é função do nível médio corrente da série temporal e pode ser adicionado ou subtraído de uma previsão que dependa apenas de nível e tendência" (Kohler et al., 2001, p. 269).

Como forma de avaliar a acurácia do modelo, ou seja, sua precisão quanto aos resultados gerados, foram adotadas os valores do Erro Médio Absoluto (MAE), representado, de acordo com Tubino (2009), pela fórmula:

$$
\mathrm{MAE}=\frac{1}{n} \sum_{i=1}^{n}\left|x_{i}-x\right|
$$

Além do MAE, de acordo com Tubino (2009), uma outra forma de avaliar os erros inerentes a previsão é a Média do Erro Absoluto Percentual (MAPE, do inglês Mean Absolute Percentage Error). Seu valor é muito importante na interpretação do modelo e mede o erro em porcentagem. A expressão utilizada com esta variável é representada por:

$$
\text { MAPE }=\frac{\sum\left(\frac{\left|\mathrm{e}_{\mathrm{t}}\right|}{\mathrm{X}_{\mathrm{t}}} * 100\right)}{\text { n.de medidas }}
$$

Onde: Xt são os dados, et são os ajustes e n é o número de observações. Quanto menor o MAPE melhor é o ajuste (Tubino, 2009). Outra medida é o Erro Quadrático Médio ou Mean Squared Error (MSE), expressa pela equação:

$$
\mathrm{MSE}=\frac{\sum \mathrm{e}_{\mathrm{t}}^{2}}{\text { n.de medidas }}
$$


Hallak \& Pereira Filho (2011) afirmam que o MSE "é similar ao MAE, porém mais sensível aos grandes erros, por elevar as diferenças individuais ao quadrado. MSE é sempre positivo. MSE $=0$ indica simulação perfeita."

\section{Aspectos metodológicos}

\subsection{Da classificação}

O presente estudo, comporta-se, diante da metodologia empregada, como estudo de caso. Nesse sentido, tem como propósito reunir informações detalhadas e sistemáticas sobre um determinado fenômeno (Patton, 2002). É um procedimento metodológico que enfatiza entendimentos contextuais e ainda na dinâmica do contexto real (Llewellyn \& Northcott, 2007).

Envolve ainda a ampliação do conhecimento (Gil, 2007). Martins (2008, p. 11) afirma que "mediante um mergulho profundo e exaustivo em um objeto delimitado, o estudo de caso possibilita a penetração em uma realidade social, não conseguida plenamente por um levantamento amostral e avaliação exclusivamente quantitativa". Do ponto de vista dos objetivos, é possível enquadrar a presente pesquisa como exploratória, que, para Gil (2007) proporciona maior familiaridade com o contexto e seus problemas, com o intuito de deixa-lo mais notório.

\subsection{Das etapas da pesquisa}

O estudo foi aplicado em uma indústria de polpa de frutas, localizada na Região Integrada de Desenvolvimento-RIDE em Pernambuco. A empresa forneceu dados históricos para que a previsão da demanda fosse executada com vistas ao segundo semestre do ano de 2017. Vale salientar que a empresa visa implementar conceitos lean em sua planta, adaptando as operações sob a filosofia.

Assim, com auxílio do software Microsoft Excel $^{\circledR}$, os dados foram obtidos para análise. Num segundo momento, foi-se comparado os ganhos acarretados pela previsão da demanda com as mudanças implementadas pela gestão. Logo, sob a ótica lean, observou-se a minimização de desperdícios no local, evidenciando-os em forma de comparativo com dados antes e depois da gestão orientada pela previsão da demanda.

Portanto, as seguintes etapas foram cumpridas:

i. Apresentação da proposta: nesta fase inicial, pôde-se apresentar aos gestores locais o propósito da pesquisa e os potenciais ganhos que a empresa poderia obter gerenciando os insumos de acordo com a demanda prevista; 
ii. Coleta de dados: para tal, dados históricos de vendas do período de 2014, 2015, 2016 e primeiro semestre de 2017 foram obtidos por meio de planilhas da empresa;

iii. Tratamento dos dados: nesta etapa, os dados inerentes ao segundo semestre dos últimos três anos (2014 a 2016) foram introduzidos na planilha do Microsoft Excel ${ }^{\circ}$ para geração da previsão da demanda para segundo semestre de 2017;

iv. Cálculo da previsão: o modelo de Holt-Winters aditivo foi aplicado e os resultados foram analisados pela empresa, que os validou;

v. Comparação dos cenários: esta atividade, discutida detalhadamente na próxima sessão, visou evidenciar o comportamento da empresa em termos de custos, estoques, qualidade e outros mais, antes da previsão e depois da mesma.

\section{Resultados e discussão}

\subsection{Cenário inicial}

A partir dos dados históricos, pôde-se perceber que as vendas dos produtos fabricados na empresa obedecem a características sazonais, onde no segundo semestre, nos meses de novembro e dezembro há uma alta nas vendas, conforme verificado no Gráfico 1.

Gráfico 1 - Comportamento de vendas - segundo semestre (2014 a 2016)

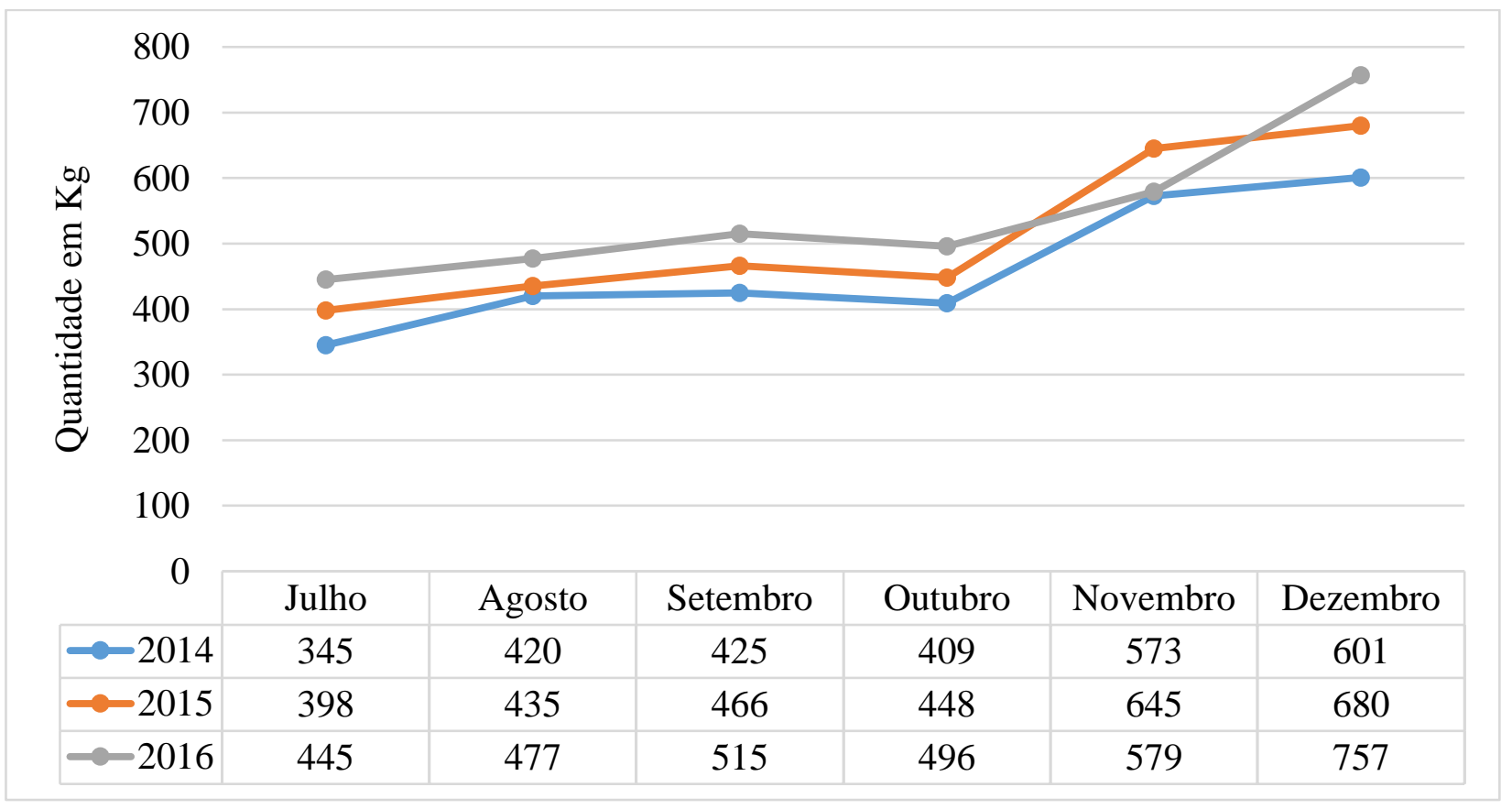

Fonte: Dados da empresa (2014 a 2016) 
Outro conjunto de dados obtidos para análise, cedido pela empresa, foram relacionados ao nível de estoque (em kg) no período do segundo semestre dos anos de 2014 a 2016, além de seus respectivos custos médios aproximados em relação a armazenagem e perdas por validade, evidenciados na Tabela 1.

Tabela 1 - Níveis de estoque e custos (2014 a 2016)

\begin{tabular}{cccccc}
\hline Mês & $\mathbf{2 0 1 4}$ & $\mathbf{2 0 1 5}$ & $\mathbf{2 0 1 6}$ & Total & Custo total médio \\
\hline Julho & 34 & 41 & 44 & 119 & $\mathrm{R} \$ 1.916,00$ \\
\hline Agosto & 57 & 21 & 17 & 95 & $\mathrm{R} \$ 1.510,00$ \\
\hline Setembro & 29 & 35 & 8 & 72 & $\mathrm{R} \$ 1.408,00$ \\
\hline Outubro & 23 & 19 & 21 & 63 & $\mathrm{R} \$ 1.246,00$ \\
\hline Novembro & 30 & 47 & 55 & 132 & $\mathrm{R} \$ 2.002,00$ \\
\hline Dezembro & 54 & 61 & 42 & 157 & $\mathrm{R} \$ 1.896,00$ \\
\hline Total & 227 & 224 & 187 & 638 & $\mathrm{R} \$ 9.978,00$
\end{tabular}

Fonte: Dados da empresa (2014 a 2016)

Como nota-se na Tabela 1, a empresa possuiu um alto custo com produtos acabados (polpas) em estoque. Além disso, considerando o ano 1 (2014) ao ano 3 (2016), a mesma apresentou alto investimento em estoque de insumos, como mostrado na Gráfico 2.

Gráfico 2 - Investimento médio por ano em insumos

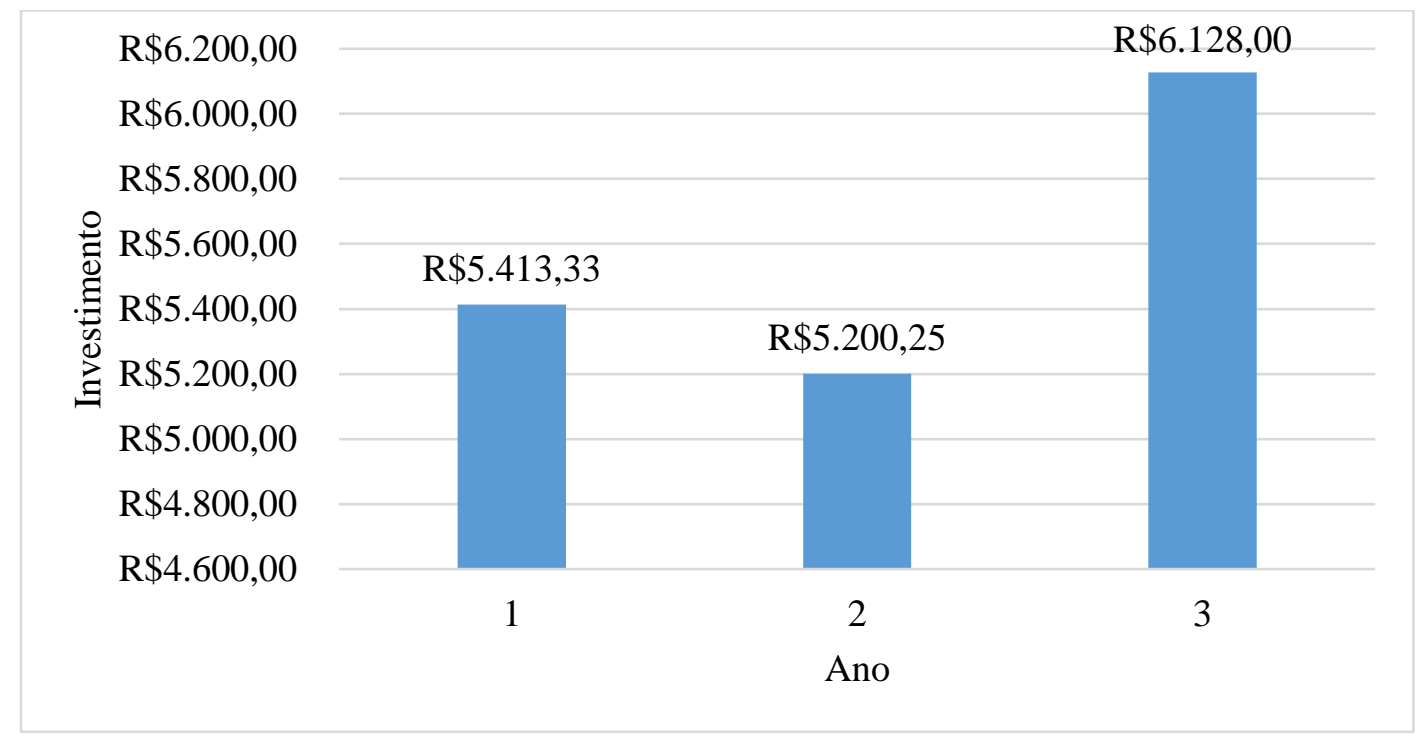

Fonte: Dados da empresa (2014 a 2016) 
Os investimentos em insumos para fabricação das polpas evoluíram cerca de 13\% nos últimos anos. Além disso, nota-se que não há planejamento quanto a esse tipo de compra, pois não faz-se nenhum tipo de previsão de vendas. Ou seja, há apenas a ideia de que o que será investido é suficiente para suprir as necessidades da empresa, sem prejudicar a receita da mesma.

\subsection{Definição da previsão de demanda}

Como as séries temporais formadas pelos dados da empresa apresentam tendência linear e sazonalidade, simultaneamente, a partir do modelo Holt-Winters para Efeitos Sazonais Aditivos, pôdese estimar as vendas para o segundo semestre de 2017, utilizando como dados de entrada os valores históricos registrados pela empresa entre 2014 e 2016. A previsão para o segundo semestre de 2017 em relação aos anos anteriores é registrada no Gráfico 3.

Gráfico 3 - Previsão da demanda para segundo semestre de 2017

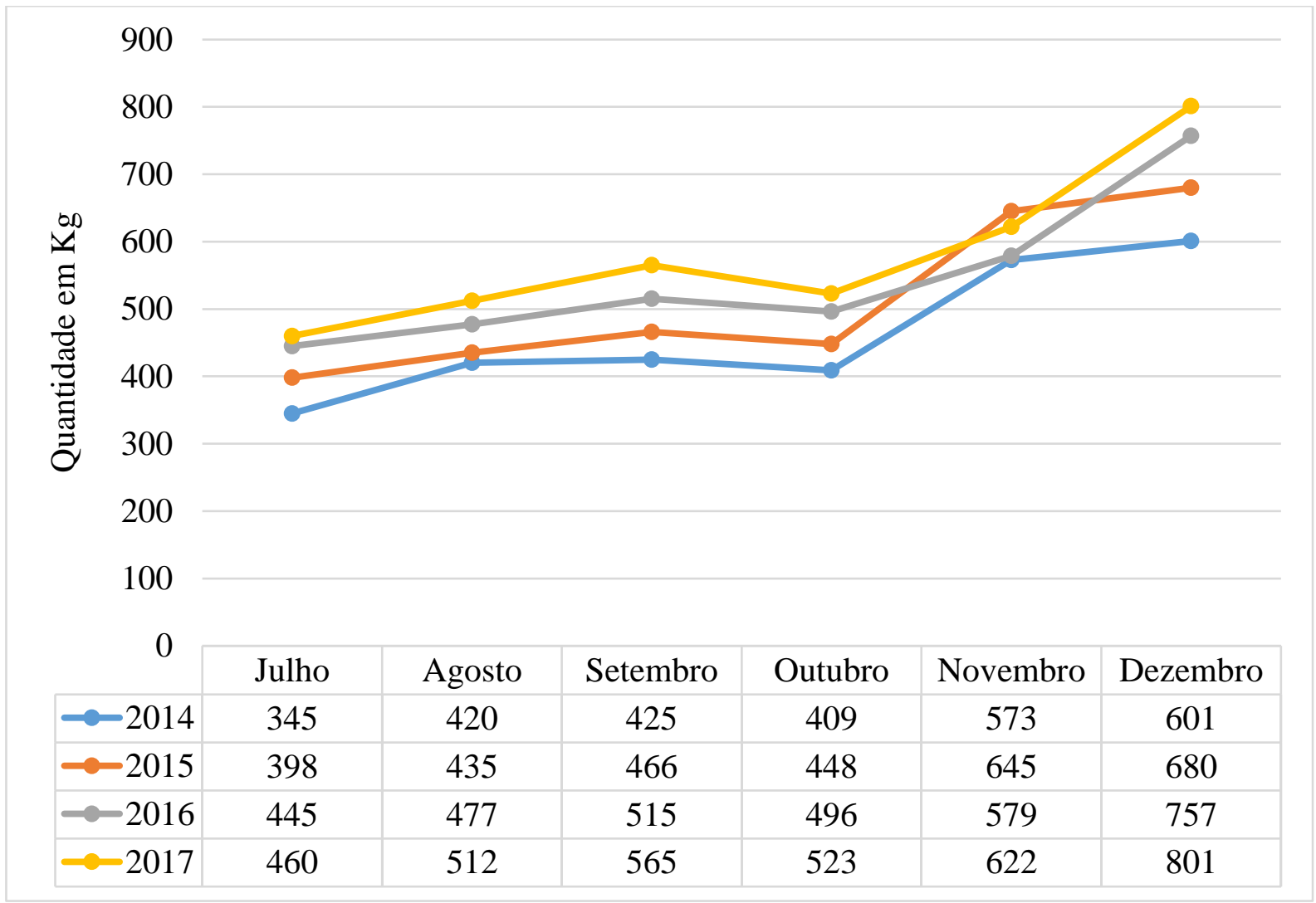

Fonte: Dados da pesquisa (2017)

A previsão conforme o modelo aditivo, apresenta comportamento previsível, quando comparado aos registros passados dos anos de 2014, 2015 e 2016. De acordo com os cálculos, o resultado é 
satisfatório, tendo em vista as estatísticas de teste e ajuste considerados pelo método, conforme Tabela 2.

Tabela 2 - Estatística de teste para previsão

\begin{tabular}{ccccc}
\hline Modelo & $\mathrm{R}^{2}$ & $\mathrm{MAD}$ & $\mathrm{MSE}$ & MAPE \\
\hline Aditivo & 0,9513 & 07,2003 & 21,5501 & $12,65 \%$
\end{tabular}

Fonte: Dados da pesquisa (2017)

Analisando as estatísticas informadas pelo software, é possível afirmar, de acordo com o coeficiente de determinação $\left(R^{2}\right)$, que $95,13 \%$ do valor é explicado pelo modelo escolhido. Trata-se de uma forma de avaliar a qualidade do ajuste do modelo. Basicamente, este valor indica quanto o modelo foi capaz de explicar os dados coletados e analisados. Para os dados, foram considerados o $\alpha=0,6, \beta=$ 0,5 e $\gamma=0,4$.

Por outro lado, analisando os erros do tipo Média Aritmética dos Desvios Absolutos em Porcentuais (MAPE), que é capaz de avaliar a magnitude do erro com relação à série histórica e a Média Absoluta dos Erros (MAD), percebe-se que são baixos, representando a precisão da previsão, com um MAD de aproximadamente $7,20 \mathrm{~kg}$ de produto acabado que corresponde a uma média percentual absoluta dos erros de 12,65\%. Ademais, a Média dos Quadrados dos Erros (MSE) apresentou um valor satisfatório, igual a $21,5501 \mathrm{~kg}$ de produto.

Assim, pôde-se comparar o que foi previsto com o que realmente ocorreu no segundo semestre de 2017, visualizado no Gráfico 4.

Gráfico 4 - Previsto versus realizado em 2017

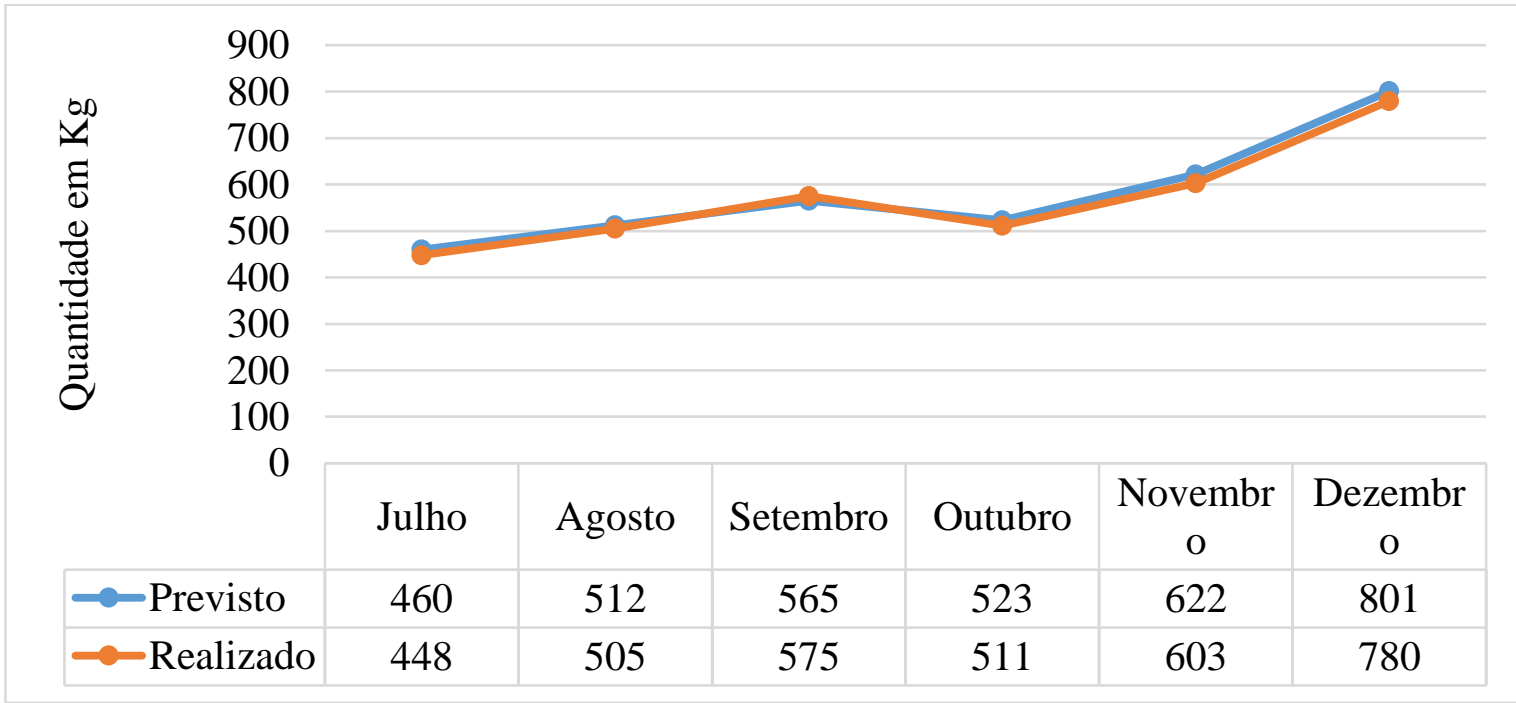

Fonte: Dados da pesquisa (2017) 
Conforme Gráfico 4, nota-se que a previsão foi bem próxima do que realmente aconteceu, com apenas um registro de valor realizado maior que previsto, indicado no mês de setembro.

\subsection{Cenário posterior}

Como a gestão local pôde tomar decisões com base na previsão realizada, somada ao fato de que há um desejo de tornar o processo enxuto, sem desperdícios, pode-se analisar alguns aspectos de melhoria ao cumprimento deste objetivo.

O primeiro fato observado foi a redução de estoque de produtos acabados, um dos desperdícios citados pela filosofia lean. Tal minimização foi ocasionada justamente pela adoção de práticas que evitam o excesso de estoque, tanto do tipo produto acabado, como de insumos ou matérias prima. Conforme mostrado na Tabela 3, o nível de estoque é bem menor que de períodos passados, quando observado a média dos anos passados com o valor obtido em 2017.

Tabela 3 - Nível de estoque (segundo semestre 2017)

\begin{tabular}{cccccc}
\hline Mês & 2014 & 2015 & 2016 & Média & 2017 \\
\hline Julho & 34 & 41 & 44 & 40 & 15 \\
\hline Agosto & 57 & 21 & 17 & 32 & 10 \\
\hline Setembro & 29 & 35 & 8 & 24 & 12 \\
\hline Outubro & 23 & 19 & 21 & 21 & 15 \\
\hline Novembro & 30 & 47 & 55 & 44 & 21 \\
\hline Dezembro & 54 & 61 & 42 & 52 & 36 \\
\hline Total & 227 & 224 & 187 & 213 & 109
\end{tabular}

Fonte: Dados da pesquisa (2017)

Isto posto, pode-se analisar uma queda relevante no quantitativo de inventário na empresa, cerca de $50 \%$, o que configura uma prática consoante à filosofia lean, representando ainda um ganho em redução de custo com estoque e perda por validade, visto na Tabela 4. 
Tabela 4 - Custo com estoque (segundo semestre 2017)

\begin{tabular}{ccc} 
Mês & $\begin{array}{c}\text { Registro } \\
(\mathrm{Kg})\end{array}$ & Custo total \\
\hline Julho & 15 & $\mathrm{R} \$ 655,00$ \\
\hline Agosto & 10 & $\mathrm{R} \$ 523,00$ \\
\hline Setembro & 12 & $\mathrm{R} \$ 416,00$ \\
\hline Outubro & 15 & $\mathrm{R} \$ 403,00$ \\
\hline Novembro & 21 & $\mathrm{R} \$ 775,00$ \\
\hline Dezembro & 36 & $\mathrm{R} \$ 890,00$ \\
\hline Total & 109 & $\mathrm{R} \$ 3.662,00$ \\
\hline
\end{tabular}

Fonte: Dados da pesquisa (2017)

Outro benefício observado, após a previsão, foi a redução de custo com investimento em matéria prima, tendo em vista que, a partir dos dados projetados, a gestão local pôde planejar melhor esse tipo de gasto. Comparando-se com a média investida nos anos anteriores, a empresa apresentou investimento controlado em insumos, conforme Gráfico 5.

Gráfico 5 - Investimento em insumos

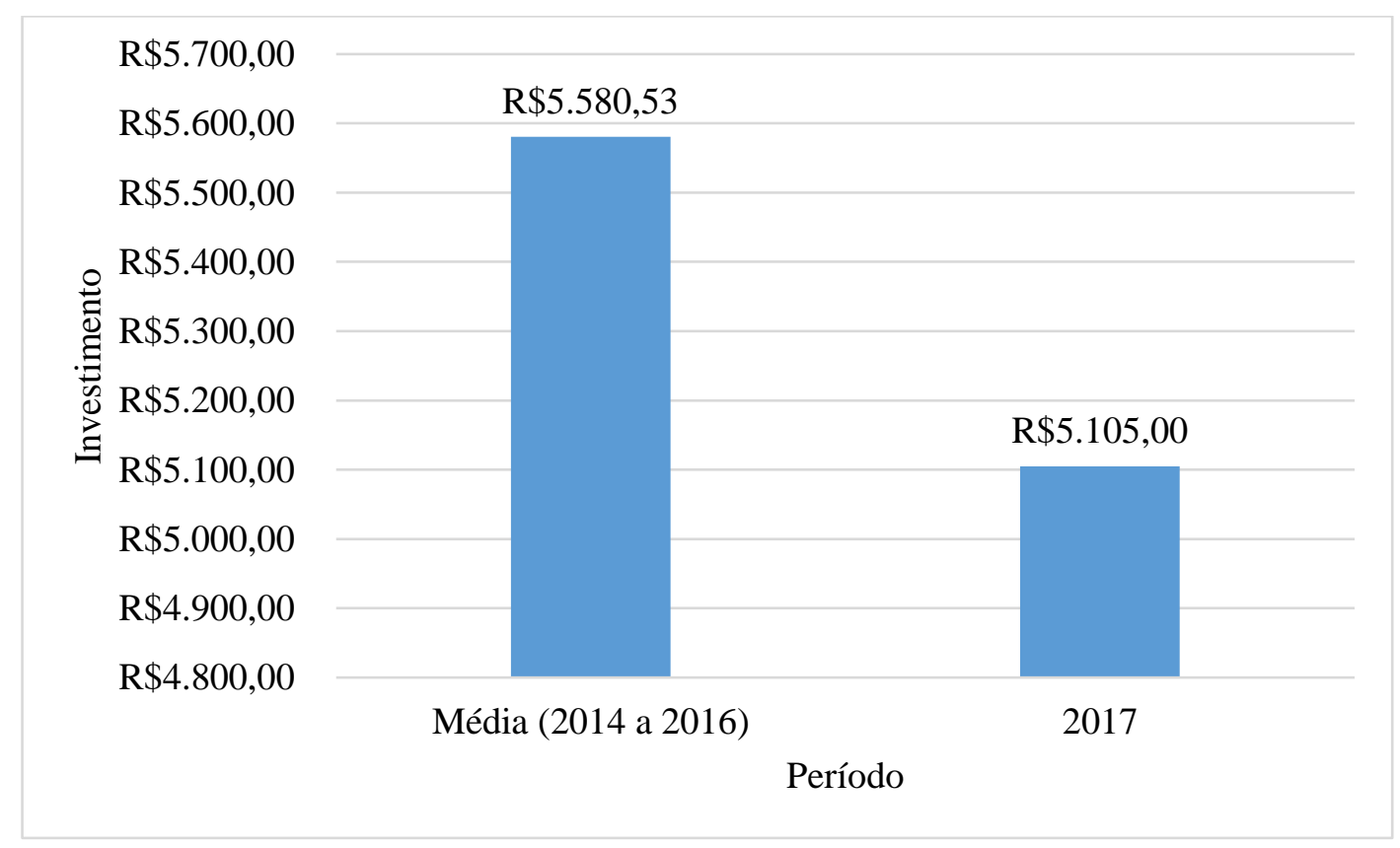

Fonte: Dados da empresa (2017)

Ou seja, comprova-se que, quando aplicada a técnica de previsão da demanda, a gestão da empresa pode planejar melhor suas aquisições, determinar investimentos em ativos, contratação de 
mão de obra, ampliação da capacidade produtiva e ainda, estimar lucro da mesma. Um cenário percebido pela gestão da empresa, diz respeito ao melhor emprego dos equipamentos na fabricação das polpas. De acordo com ela, o desperdício de espera, causado pela falta de planejamento de utilização dos equipamentos industriais, formando filas que proporcionavam altas taxas de utilização dos mesmo, diminuiu em decorrência da elaboração de um plano de produção, onde, seguindo o comportamento da previsão, as ordens de produção eram emitidas, evitando assim, produção em excesso.

Esta observação reforça o fato de que o sistema lean é capaz de enfatizar o fluxo de materiais que devem ser submetidos ao processamento, quando este for realmente necessário. Nesse caso, a produtividade, gerada pelo bom uso das máquinas e com operações bem dimensionadas, é fator determinante para manutenção do nível de competitividade da empresa.

Ademais, do ponto de vista da filosofia lean, os desperdícios não resumem-se apenas a excesso de estoque, espera ou ainda produção desnecessária. Porém, com a utilização de técnicas de previsão de demanda, ações que, indiretamente ou não, contribuem para difusão e adesão ao pensamento enxuto podem ser notadas e praticadas continuamente.

Outro ponto importante a salientar é que, as melhorias devem ser contínuas, ou seja, a empresa não deve limitar-se a ganhos imediatos. É fundamental a manutenção de práticas que, de fato, agregaram valor ao processo e que, portanto, contribuirão para o desenvolvimento da empresa como um todo. O envolvimento dos funcionários é de extrema importância também na disseminação da filosofia lean, sendo uma oportunidade de aprimoramento constante da equipe de trabalho, capacitando-os cada vez mais sob a ótica enxuta na busca pela perfeição.

\section{Considerações finais}

A filosofia lean tem como base a harmonia entre a combinação de técnicas de gestão e os equipamentos, a fim de produzir mais com menos recursos. Isto significa que, para cumprimento desse propósito, o pensamento enxuto busca assegurar processos estáveis, com alto nível de padronização e com qualidade garantida. Envolve a melhoria contínua, destacando-se em criar valor na perspectiva do cliente final, eliminando assim desperdícios ao longo do processo.

Por outro lado, a previsão da demanda pode atuar como suporte à implementação de uma filosofia lean na indústria, conforme proposto neste trabalho. Ficou evidenciado que uma empresa que visualiza cenários futuros, projetados pelos métodos confiáveis de previsão de demanda, podem usufruir de vários benefícios. O estudo comprovou que, quando aplicada a técnica de previsão, a gestão da empresa pode planejar melhor suas aquisições, determinar investimentos em ativos, contratação de mão de obra, ampliação da capacidade produtiva e ainda, estimar lucro da mesma. 
Todas as vantagens conseguidas com melhor planejamento dos aspectos que envolvem a manufatura, contribuem diretamente para a maior competitividade da organização frente as demais no mercado atual. Assim, qualquer empresa que almeja implementar modelos de gestão modernos, enfatizará, como o lean, o fluxo de materiais que devem ser submetidos ao processamento, quando este for realmente necessário. Nesse caso, a produtividade, gerada pelo bom uso das máquinas e com operações bem dimensionadas, é fator determinante para manutenção do nível de competitividade da empresa.

Logo, o objetivo do trabalho foi atingido, demonstrando, a partir de um caso real, que a previsão da demanda pode auxiliar a empresa na modernização de sua gestão, associando-a com práticas modernas e consistentes, como a filosofia lean. Ademais, é notório os ganhos que a empresa teve a partir de um planejamento de produção mais acurado, com vistas a reduzir custos oriundos, principalmente, de desperdícios no processo de fabricação das polpas.

Recomenda-se, para o caso específico da empresa estudada, a aplicação de ferramentas lean, como o Mapeamento do Fluxo de Valor (MFV) para reunir informações mais detalhadas sobre o processo, inclusive acerca de tempo de ciclo, lead time, estoques intermediários e outras mais. Além disso, como sugestão para trabalhos futuros, cita-se a aplicação de técnicas de previsão de cunho qualitativas e também das outras quantitativas, como a regressão, média móvel exponencial e outras disponíveis na literatura, em ambientes industriais, para comparar os ganhos obtidos em termos de eliminação e/ou redução de desperdícios e melhoria de produtividade e qualidade, bases do pensamento enxuto.

\section{Referências}

Alefari, M., Salonitis, K., \& Xu, Y. (2017) The role of leadership in implementing lean manufacturing. Procedia CIRP, 63, $756-761$.

Ballou, R. H. (2006) Gerenciamento da cadeia de suprimentos: planejamento, organização e logística empresarial. Bookman.

Bayat, H., \& Dadashzadeh, M. (2017) The impact of organizational factors on implementation outcomes of lean manufacturing. Journal of Business \& Economics Research (JBER), 15(2), 33-44.

Begam, M. S., Swamynathan, R. \& Sekkizhar, J. (2014). A brief overview of current trend on lean management practices in manufacturing industries. Annals of the Faculty of Engineering Hunedoara, 12(2), 35.

Bose, J-H., Flunkert, V., Gasthaus, J., Januschowski, T., Lange, D., Salinas, D., Schelter, S., Seeger, M., \& Wang. (2017) Probabilistic Demand Forecasting at Scale. Proceedings of the VLDB Endowment, 10(12).

Corrêa, H. L., Gianesi, I. G. N., \& Caon, M. (2012) Planejamento, programação e controle da produção: MRP II/ERP conceitos, uso e implantação: base para SAP, Oracle Applications e outros softwares integrados de gestão. São Paulo: Atlas.

Drew, J., McCallum, B., \& Roggenhofer, S. (2016). Journey to lean: Making operational change stick. Springer. 
Forbes, L. H., \& Ahmed, S. M. (2010). Modern construction: Lean project delivery and integrated practices. Boca Raton, FL: CRC Press.

Gil, A. C. (2007). Métodos e Técnicas de Pesquisa Social. 5 ed. São Paulo: Atlas.

Gobinath, S., Elangovan, D. \& Dharmalingam, S. (2015). Lean Manufacturing Issues and Challenges in Manufacturing Process-A Review. International Journal of ChemTech Research, . 8(1), 44-51.

Hallak, R., \& Pereira Filho, A, J. (2011). Metodologia para análise de desempenho de simulações de sistemas convectivos na região metropolitana de São Paulo com o modelo ARPS: sensibilidade a variações com os esquemas de advecção e assimilação de dados. Revista Brasileira de Meteorologia, 26(4), 591-608.

Henning, E., Alves C. C., \& Konrath, A.C. (2010) Previsão de vendas de rodízios para móveis em uma empresa de médio porte. In: ICPR AMERICAS - 5th Americas International Conference on Production Research, Bogotá, Colômbia, 2010. Proceedings... Bogotá.

Hyndman, R., Koehler, A., Ord, J., \& R. Snyder. (2008) Forecasting with Exponential Smoothing: The State Space Approach. Springer, 1 ed.

Kerkkânen, A., Korpela, J., \& Huiskonen, J. (2009) Demand forecasting errors in industrial context: Measurement and impacts. International Journal of Production Economics, 118, 43-48.

Klassen, R., \& Flores, B. (2001) Forecasting practices of Canadian Firms: Survey Results and comparisons, International Journal of Production Economics. 70(2), 163-174.

Koehler, A. B., Snyder, R. D., \& Ord, J. K. (2001) Forecasting Models and Prediction Intervals for the Multiplicative Holt-Winters Method. International Journal of Forecasting, 17(2), 269-286.

Lee, C. K., Song, H. J., \& Mjelde, J. W. (2008) The forecasting of international expo tourism using quantitative and qualitative techniques. Tourism Management, 29(6), p. 1084-1098.

Llewellyn, S., \& Northcott, D. (2007) The "singular view" in management case studies qualitative research in organizations and management. An International Journal, 2(3), 194-207.

Lopes, R. B., Freitas, F. \& Sousa, I. (2015). Application of Lean Manufacturing Tools in the Food and Beverage Industries. Journal of Technology Management \& Innovation. 10(3).

Lustosa, L., Mesquita, M. A., Quelhas, O., \& Oliveira, R. (2008) Planejamento e controle da Produção. Rio de Janeiro: Elsevier.

Makridakis, S., Wheelwright, S., \& Hyndman, R. J. (1998) Forecasting Methods and Applications. 3. ed. New York: John Wiley \& Sons.

Martins, G. A. (2008) Estudo de caso: uma reflexão sobre a aplicabilidade em pesquisas no Brasil. Revista de Contabilidade e Organizações, 2(2), 9-18.

Modi, D. B., \& Thakkar, H. (2014). Lean Thinking: Reduction of Waste, Lead Time, Cost through Lean Manufacturing Tools and Technique. International Journal of Emerging Technology and Advanced Engineering, 4(3).

Moreira, D. A. (2001) Administração da produção e operações. São Paulo: Pioneira Thomson Learning; Pająk E. (2013). Principles and methods of lean production, IBUK Libra, Konin.

Panizzolo, R., Garengo, P., Sharma, M. K., \& Gore, A. (2012) Lean manufacturing in developing countries: Evidence from Indian SMEs. Production Planning \& Control, 23(10), 769-788.

Patton, M. G. (2002) Qualitative Research and Evaluation Methods, 3 ed. Thousand Oaks, CA: Sage.

Sabadka, D., Molnar, V., \& Fedorko, G. (2017) The Use of Lean Manufacturing Techniques - SMED Analysis to Optimization of the Production Process. Advances in Science and Technology Research Journal, 11(3), 187-195.

Santos, P. V. S. (2017). A Indústria Vinícola No Vale Do São Francisco e As Estratégias de Inserção No Mercado Nacional: Uma Revisão Bibliográfica. Future Studies Research Journal, 9, 39-68.

Santos, P. V. S., \& Lima, N. V. M. (2018) Fatores de Impacto para Sobrevivência de Micro e Pequenas Empresas (MPEs). Revista Livre De Sustentabilidade E Empreendedorismo, 3, 54-77.

Shiver, J. M., \& Eitel, D. (2010) Optimizing Emergency departmentthroughput: Operations management solutions for health care decisionmakers. New York: Taylor \& Francis Group.

Slack, N., Jones, A. B. \& Johnston, R. (2013). Princípios de administração da produção. São Paulo: Atlas.

Sokovic, M., Pavletic, D., \& Fakin, S. (2005). Application of Six Sigma methodology for process design. Journal of Materials Processing Technology. 162, 777-783. 
Trojanowska, J., Kolińska, K., \& Koliński, A., (2011), Using the Lean Tools in Production Enterprises as an Effective Way to Win With Economic Crisis, Problemy Zarzqdzania, 9(1), 34- 52.

Tubino, D. F. (2009). Planejamento e controle da produção: teoria e prática (2a ed.). São Paulo: Atlas. Wilson, L (2010) How to Implement Lean Manufacturing, 1 st Edition McGraw Hill, New York.

Władysiak, R. (2006). Reengineering of precision castings production system. Arch. Foundry Eng. 6(19), 413-424.

Womack, J., Jones, D. T., \& Roos, D. (2004) A máquina que mudou o mundo. São Paulo: Campus.

Zakaria, N. H., Zuki, N. M., Mohamed, N., Ab Rahid, M. F. F. \& Rose, A. N. M. (2017). Lean manufacturing implementation in reducing waste for electronic assembly line: MATEC Web of Conferences, 90. 